\title{
Entrée en vigueur des directives révisées sur le diagnostic de la mort
}

\author{
Jürg Steigera, Michelle Salathéb \\ a Prof Dr méd., Président de la Commission Centrale d'Ethique (CCE) de I'ASSM; ' lic. en droit, secrétaire générale adjointe de I'ASSM
}

La Loi fédérale sur la transplantation d'organes, de tissus et de cellules (LTx) a été révisée et entrera en vigueur le 15 novembre 2017. Suite à la révision de la LTx, une révision des directives de l'ASSM «Diagnostic de la mort dans le contexte de la transplantation d'organes» s'est imposée. Cet article résume les principales nouveautés.

Les conditions requises pour un prélèvement d'organes sont réglées dans la Loi sur la transplantation d’organes, de tissus et de cellules (LTx). Pour définir le critère de la mort, la loi se fonde sur la définition neurologique de la mort, selon laquelle une personne est décédée lorsque toutes les fonctions du cerveau, y compris celles du tronc cérébral, ont subi un arrêt irréversible. Le législateur laisse aux experts médicaux le soin de définir comment la mort est constatée lege artis. L’Ordonnance concernant la TLx renvoie aux directives «Diagnostic de la mort et préparation du prélèvement d'organes en vue de la transplantation d'organes» de l'ASSM; les chapitres correspondants ont force de loi.

La version révisée de la LTx devrait entrer en vigueur le 15 novembre 2017. La révision a notamment tenu compte de deux préoccupations majeures de l'ASSM, sources de confusion dans la pratique clinique. Celles-ci concernent les questions suivantes:

1. Quel est le moment adéquat pour parler du don d'organes avec les proches?

2. À quelles mesures médicales préliminaires les proches peuvent-ils consentir si le patient ne s'est pas exprimé lui-même à ce sujet?

Suite à la révision de la LTx, une révision des directives de l'ASSM concernant le diagnostic de la mort, datant de 2011, s'est imposée. Comme toutes les directives médico-éthiques de l'ASSM, les directives révisées ont été élaborées par une sous-commission interprofessionnelle; celle-ci est dirigée par le Prof. Jürg Steiger de Bâle. Le projet de texte a été discuté avec des experts choisis et mis en consultation avant d'être approuvé par les organes de l'ASSM (Commission Centrale d'Ethique, Comité de direction et Sénat). Ces directives entrent en vigueur le 15 novembre 2017.
Les points centraux suivants des directives de 2011 demeurent inchangés:

- les conditions du critère de la mort (signes cliniques);

- l'examen par deux médecins spécialistes (principe de surveillance mutuelle), pas d'intervalle de temps;

- la nécessité de réaliser des examens techniques complémentaires uniquement lorsque les conditions requises pour l'examen clinique ne sont pas remplies (c'est-à-dire lorsque l'examen clinique des nerfs cérébraux est impossible ou que l'éventualité de facteurs potentiellement réversibles ne peut être exclue).

- pas de prélèvement d'organes chez les nouveau-nés.

\section{Aperçu des principaux points révisés}

À quel moment la question du don d'organes

peut-elle être abordée avec les proches?

L'ancien texte de loi ne précisait pas à quel moment le thème du don d'organes peut être abordé avec les proches. Cette question est clarifiée avec les nouvelles dispositions. La séparation claire entre la décision d'interrompre un traitement de maintien en vie et la décision du don d'organes est essentielle. Les indications de traitements intensifs et de mesures d'urgence ne doivent pas être influencées par l'éventualité d'un don d'organes. Le consentement au prélèvement d'organes et aux éventuelles mesures médicales préliminaires ne doit être demandé qu'après avoir décidé d'interrompre les thérapies de maintien en vie. Les entretiens concernant la possibilité du don d'organes peuvent néanmoins être menés avant la constatation de la mort; souvent les proches abordent eux-mêmes ces sujets. 
Quelles sont les conditions requises pour entamer le processus de la constatation de la mort?

La révision des directives de l'ASSM ne tient pas uniquement compte des adaptations de la TLx révisée, mais également des enseignements tirés de la pratique. La principale nouveauté est la description, en annexe C, des conditions cliniques devant être remplies avant de pouvoir établir le diagnostic de la mort, contribuant ainsi à améliorer la sécurité du processus.

\section{Qui est habilité à constater la mort?}

Les directives révisées décrivent avec plus de précisions les exigences posées aux médecins qui constatent la mort. Elles imposent qu'au moins un des deux spécialistes ait procédé à un diagnostic de mort cérébrale sous supervision chez au moins cinq patients.

Quelles mesures préliminaires peuvent être adoptées déjà avant la mort et quel est le rôle des proches?

La question de savoir si des mesures médicales préliminaires peuvent être appliquées avant la mort et lesquelles revêt une importance majeure dans la pratique, car la plupart des patients meurent à l'unité de soins intensifs et non pas en pleine thérapie. A cela s'ajoute que seule une petite partie des patients détiennent une carte de donneur d'organes, la question du don d'organes restant alors ouverte. Toutefois, afin de maintenir la fonction vitale des organes jusqu'au prélèvement, des mesures médicales préliminaires doivent être adoptées. L'ancienne LTx ne précisait pas si les proches pouvaient consentir à des mesures préliminaires avant la mort lorsque le patient concerné ne s'est pas exprimé lui-même à ce sujet. La nouvelle LTx stipule que les proches habilités à représenter le patient peuvent - à quelques exceptions près - consentir aux mesures préliminaires, même s'ils ne savent pas comment le patient aurait décidé. En complément à la LTx, les directives présentent le processus décisionnel com- plexe dans un flowchart et récapitulent dans une «liste négative» les mesures auxquelles les proches ne peuvent pas consentir. Il s'agit de la pose de canules artérielles et de la réalisation d'une réanimation mécanique.

Comment gérer les situations, où des proches refusent un prélèvement d'organes par conviction personnelle malgré l'existence d'une carte de donneur?

Cette situation difficile pour l'équipe de soins se présente effectivement dans la pratique clinique quotidienne. Les directives renvoient à la législation qui stipule que la volonté de la personne décédée prime celle des proches. Les directives recommandent de tout mettre en œuvre pour parvenir à un consensus entre toutes les personnes concernées et de ne pas hésiter à avoir recours à un soutien éthique.

L’objectif de la version révisée des directives est de soutenir l'équipe de soins dans l'application des dispositions légales en vigueur et de lui permettre de mieux gérer les nombreuses questions éthiques soulevées. Si la constatation de la mort repose sur des faits scientifiques, elle ne revêt pas moins une dimension sociétale. Ceci se reflète dans les procédures en vigueur dans d'autres pays qui, malgré leurs similitudes fondamentales, divergent en de nombreux aspects. Pour toutes les personnes concernées, l'objectif est d'acquérir la certitude maximale qu'au moment du prélèvement d'organes le donneur est mort et la garantie qu'à tout moment le mourant, respectivement sa dépouille, est traité avec respect.

Les directives ainsi que leurs annexes et le flowchart peuven être consultés en allemand, français, italien et anglais dans la version en ligne sur le site de I'ASSM, où elles peuvent également être commandées sous forme de brochure (allemand/ français): assm.ch/directives 